\title{
Ocular Ischemic Syndrome: The Red Eye Revisited
}

\author{
Prabhu Premkumar ${ }^{2}$ \\ ${ }^{1}$ Department of Interventional Radiology, Division of Clinical \\ Radiology, Christian Medical College, Vellore, Tamil Nadu, India \\ ${ }^{2}$ Department of Vascular Surgery, Christian Medical College, \\ Vellore, Tamil Nadu, India
}

Sathya Narayanan ${ }^{1}$ Shyamkumar N. Keshava ${ }^{1}$ Vinu Moses ${ }^{1} \quad$ Aswin Padmanabhan ${ }^{1}$

\begin{abstract}
Address for correspondence Shyamkumar N. Keshava, MBBS, DMRD, DNB, FRCR, FRANZCR, Department of Interventional Radiology, Division of Clinical Radiology, Christian Medical College, Vellore, Tamil Nadu 632004, India (e-mail: aparna_shyam@cmcvellore.ac.in).
\end{abstract}

J Clin Interv Radiol ISVIR:2020;4:189-192

\begin{abstract}
Keywords

- ocular ischemic syndrome

- angiography

- balloon test occlusion

Ocular ischemic syndrome (OIS) is a vision-threatening condition due to inadequate arterial supply to the orbital contents. OIS is commonly described secondary to severe carotid artery stenosis and most often observed by ophthalmologists. However, OIS may rarely also result in an interventional radiology setup during balloon test occlusion (BTO) of the internal carotid artery. BTO is a procedure to assess for the adequacy of the circle of Willis to compensate for a permanent parent arterial sacrifice by temporarily occluding the flow in the internal carotid artery using a balloon. Here, we present a case of OIS in a patient who underwent BOT as a part of presurgical evaluation for the excision of carotid body tumor.
\end{abstract}

\section{Introduction}

Ocular ischemic syndrome (OIS) is not an uncommon ocular emergency encountered in clinical practice. Although OIS is observed by ophthalmologists due to severe ipsilateral carotid artery stenosis, it can be iatrogenically introduced by neurointerventional radiologists during carotid balloon test occlusion (BTO). The pathogenesis of OIS is inadequate collateral supply to the globe secondary to severe common or internal carotid artery (ICA) stenosis. During BTO, OIS develops due to rapid saline infusion distal to the site of occlusion. This situation creates a dilemma for the intervention radiologist regarding whether the patient has failed BTO or if it is a false-positive test. The purpose of this case report is to highlight the importance for neurointerventional radiologists to be aware of such a condition, as well as its clinical implications and the technique during BTO to avoid OIS.

\section{Case History}

A 21-year-old male patient presented with gradually increasing neck swelling on the left side since 6 months. On examination, a firm mass was palpable on the left side of the neck. Contrast-enhanced computed tomography (CT) showed an intensely enhancing lesion measuring $50 \times 60 \mathrm{~mm}$ at the

published online

23-Apr-2020
DOI https://doi.org/

10.1055/s-0040-1705267

ISSN 2457-0214. bifurcation of the left common carotid artery, with splaying and encasement of carotid arteries typical of a type III carotid body tumor ( - Fig. 1). The patient was planned for BTO followed by preoperative embolization of the external carotid artery (ECA) branches supplying the tumor.

Preprocedure work-up with bleeding parameters (prothrombin time, activated partial thromboplastin time, and platelet count) and viral marker screening was performed. The procedure was performed under conscious sedation. Continuous neurologic testing was performed by somatosensory evoked potential monitoring (SSEP).

Under aseptic precautions, right groin was accessed with a 7-Fr sheath to perform the cerebral angiography in a digital subtraction angiography suite (Artis zee Biplane system, Siemens AG). The left carotid was then cannulated using a 4-Fr vertebral glide catheter. Preprocedural left common carotid angiogram revealed extensive tumor blush in the region of carotid bifurcation from the branches of the left ECA. No tumor supply was seen from the ipsilateral ICA or right carotid vessels. Cerebral angiogram revealed no abnormality and good cross-circulation across the anterior communicating artery.

The vertebral glide catheter was exchanged over a 0.035-inch exchange-length straight-tip wire to a 7-Fr SwanGanz (Edwards LifeSciences) double-lumen balloon catheter connected to a heparinized saline infusion $(5,000 \mathrm{U} / \mathrm{L})$, 


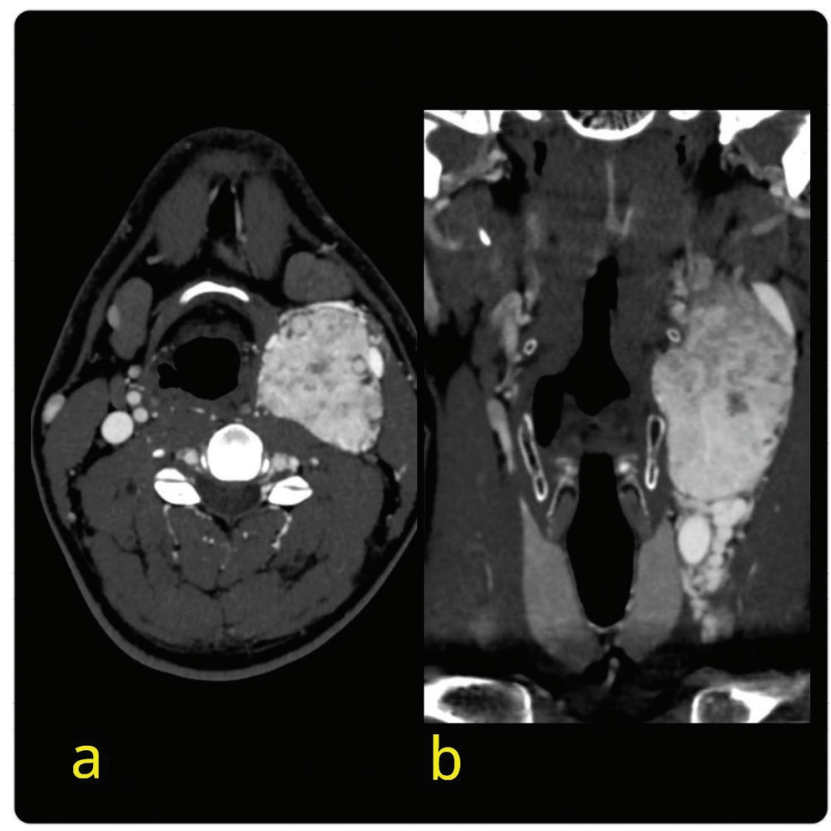

Fig. 1 A 21-year-old male presented with left-sided carotid body tumor. Contrast-enhanced computed tomography (CT) of the neck in axial (a) and coronal (b) sections reveal a left-sided carotid body tumor. Note the hyperenhancement of the mass and splaying of the internal and external carotid arteries.

approximately 40 drops per minute. The balloon was placed in the proximal left ICA beyond the carotid bulb. The baseline neurologic assessment was normal.

The Swan-Ganz balloon ( - Fig. 2) was then inflated with half-strength contrast till contrast stasis was seen in the ICA distal to the occlusion. Continuous SSEP monitoring and neurologic examination were performed. After 3 minutes

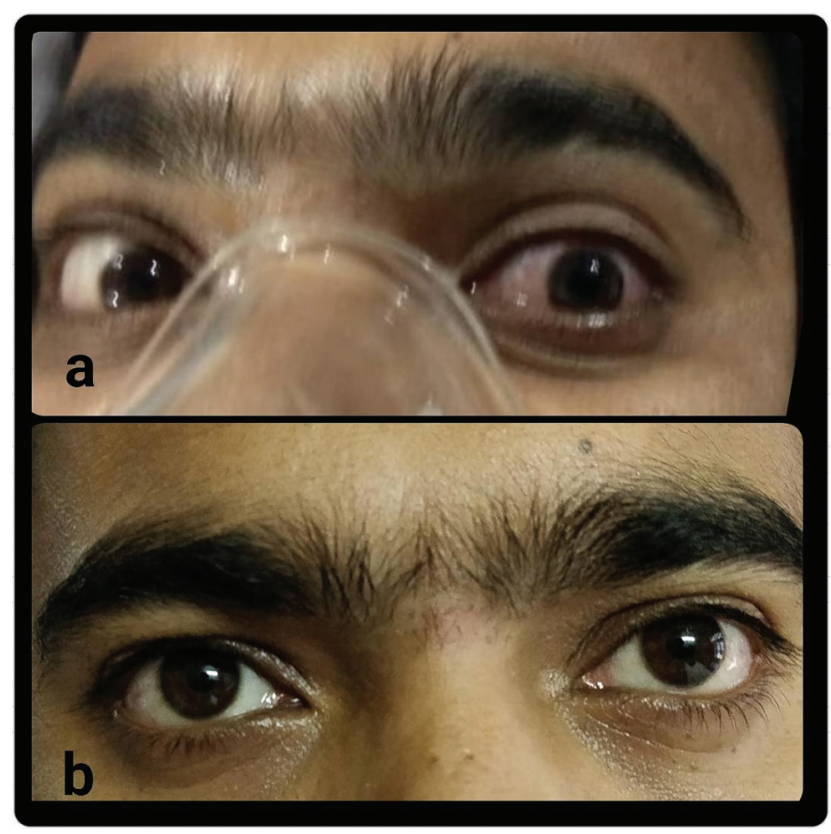

Fig. 2 A 21-year-old male presented with left-sided carotid body tumor. (a) The left eye is congested 3 minutes after balloon test occlusion of the left internal carotid artery. (b) Relief from congestion after balloon deflation. of occlusion, the patient complained of left orbital pain. On examination, the left eye (with balloon inflated) was congested ( - Fig. 2). Though his eye movements were normal, the visual acuity reduced and he was not able to count the fingers. The Swan-Ganz balloon was immediately deflated, and there was relief of pain, improvement in visual acuity, and gradual decrease in redness ( - Fig. 2). BTO was discontinued because the congestion in the left eye persisted even after 30 minutes. Following BTO, preoperative embolization of the ECA branches supplying the tumor was performed. The patient underwent successful surgical resection of the tumor.

\section{Discussion}

OIS is a well-known clinical entity in patients with severe atherosclerotic ICA or common carotid artery stenosis. It was first described by Kearns and Hollenhorst in 1963. ${ }^{1,2}$ The patients with OIS present with ipsilateral orbital pain and visual loss. The pain is dull aching and visual loss can be rapid abrupt or gradually progressive. Decreased perfusion in the ophthalmic artery due to poor blood flow beyond the occluded ICA and inadequate collaterals from the ECA and contralateral ICA contribute to the symptoms. ${ }^{2,3}$ Atherosclerosis is the most common cause of OIS., ${ }^{4,5}$ Other causes include vasculitis, dissecting aneurysms of the carotid artery, and radiotherapy for head and neck malignancies. ${ }^{5}$

In the early 1970s, Serbinenko introduced the technique of temporary balloon occlusion of the carotid artery using small endovascular balloons. ${ }^{6}$ BTO with clinical surveillance has reduced the postoperative stroke risk in comparison to unselected carotid artery sacrifice. ${ }^{7,8}$ The indications for BOT include cases in which there is a plan to sacrifice the ICA, such as giant ICA aneurysm or pseudoaneurysms, preoperative assessment of head and neck malignancies with ICA involvement, intractable hemorrhage secondary to trauma or infection or neoplasm, and carotid-cavernous fistula, which cannot be treated without parent artery occlusion. ${ }^{9}$

BTO is commonly performed with a double-lumen balloon catheter such as a 7-Fr Swan-Ganz catheter. The patient is kept under conscious sedation and continuous SSEP monitoring. After obtaining access to the ICA, the balloon is inflated with half-strength contrast just enough to occlude the ICA lumen at the level of C2. ${ }^{10}$ After confirmation of complete occlusion by injecting contrast through the contrast lumen, which will create stasis in the ICA distal to occlusion, the ICA is flushed with heparinized saline from a pressure pack maintained well above the systemic blood pressure of the patient, at approximately $300 \mathrm{~mm} \mathrm{Hg}$. Saline flow is maintained through the main lumen of the balloon catheter to avoid the stasis of blood and thromboembolic complications. ${ }^{10}$ The occlusion time is variable ranging from a few minutes to 40 minutes. During this entire period, the patient is monitored for sensorimotor level, cranial nerve status, and level of consciousness. Any change in the neurologic status during these 20 minutes or at the completion of 


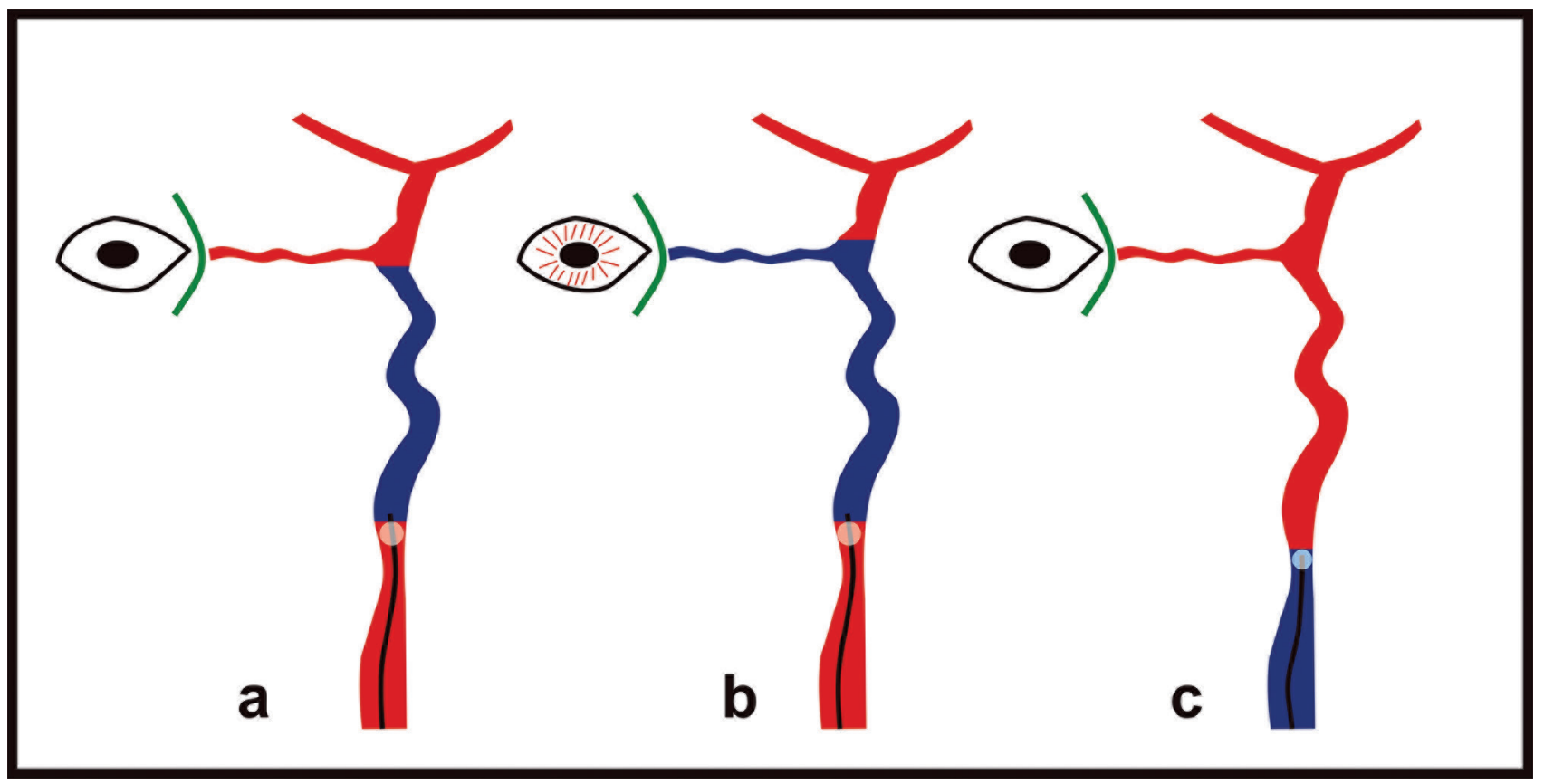

Fig. 3 (a) Slow saline flush through the double-lumen balloon occlusion catheter allows retrograde filling of the ophthalmic artery. (b) Rapid saline flush replaces the oxygenated blood in the ophthalmic artery, resulting in ocular ischemic syndrome. (c) Single-lumen balloon occlusion catheter allows retrograde filling of the ophthalmic artery.

20 minutes is considered the end point of the test. The balloon is then deflated completely and withdrawn.

OIS is one of the complications of BTO. ${ }^{4}$ This may be potentially misinterpreted as a failed BTO and affecting the management of the patient. In the index case, the orbital pain and visual loss that occurred immediately after balloon occlusion with normal SSEP tracings and sensorimotor level prompted us to consider OIS. It has been postulated that heparinized saline replaces oxygenated blood in the ipsilateral ophthalmic artery if the saline is infused at a rapid rate. ${ }^{4}$ This continuous rapid inflow of saline may also prevent retrograde filling of the ophthalmic artery with oxygenated blood from the contralateral ICA ( - Fig. 3). This will result in OIS, and the patient will develop ipsilateral orbital pain, redness, and reduction in visual acuity. ${ }^{4}$ We have performed approximately 100 cases of BTO, and our patient is the second case complaining of ipsilateral orbital pain without any other neurologic deficits and normal SSEP.

In case of OIS due to rapid saline infusion, the symptoms are likely to disappear once the rate of saline flush is reduced to a trickle (flow rate in the range of $1-10 \mathrm{~mL} / \mathrm{min}$ ) or intermittent flushing of the balloon catheter is performed.

\section{Conflict of Interest}

None.

\section{Acknowledgments}

Authors would like to thank the Department of Vascular Surgery, Christian Medical College.

\section{References}

1 Vazirani JA, Zadeng Z, Dogra MR, Gupta A. Ocular ischemic syndrome: a classical presentation of an uncommon condition. Indian J Ophthalmol 2014;62(5):658-660

2 Kearns TP, Hollenhorst RW. Venous-stasis retinopathy of occlusive disease of the carotid artery. Proc Staff Meet Mayo Clin 1963;38:304-312

3 Terelak-Borys B, Skonieczna K, Grabska-Liberek I. Ocular ischemic syndrome - a systematic review. Med Sci Monit 2012;18(8):RA138-RA144

4 Russell EJ, Goldberg K, Oskin J, Darling C, Melen O. Ocular ischemic syndrome during carotid balloon occlusion testing. AJNR Am J Neuroradiol 1994;15(2):258-262

5 Brown GC, Magargal LE. The ocular ischemic syndrome. Clinical, fluorescein angiographic and carotid angiographic features. Int Ophthalmol 1988;11(4):239-251

6 Serbinenko FA. Balloon catheterization and occlusion of major cerebral vessels. J Neurosurg 1974;41(2):125-145

7 Gonzalez CF, Moret J. Balloon occlusion of the carotid artery prior to surgery for neck tumors. AJNR Am J Neuroradiol 1990;11(4):649-652

8 Linskey ME, Jungreis CA, Yonas $\mathrm{H}$, et al. Stroke risk after abrupt internal carotid artery sacrifice: accuracy of preoperative assessment with balloon test occlusion and stable xenon-enhanced CT. AJNR Am J Neuroradiol 1994;15(5):829-843

9 American Society of Interventional and Therapeutic Neuroradiology. Carotid artery balloon test occlusion. AJNR Am J Neuroradiol 2001;22(8, Suppl):S8-S9

10 Sudhakar KV, Sawlani V, Phadke RV, Kumar S, Ahmed S, Gujral RB. Temporary balloon occlusion of internal carotid artery: a simple and reliable clinical test. Neurol India 2000;48(2):140-143 